\title{
UCLA
}

\section{Paroles gelées}

\section{Title}

"La répétition de l'Histoire" . La mise en scène narrative de la commémoration dans Revoir Nevers de Roger Magini

\section{Permalink}

https://escholarship.org/uc/item/5×3433jx

\section{Journal}

Paroles gelées, 29(0)

\section{ISSN}

\section{4-7264}

\section{Author}

Kühn, Marion

\section{Publication Date}

2015

\section{DOI}

10.5070/PG7290024364

\section{Copyright Information}

Copyright 2015 by the author(s). All rights reserved unless otherwise indicated. Contact the author(s) for any necessary permissions. Learn more at https://escholarship.org/terms

Peer reviewed 


\title{
"La répétition de l'Histoire": la mise en scène narrative de la commémoration dans Revoir Nevers de Roger Magini
}

\author{
Marion Kühn \\ Université Laval, Centre de recherche interuniversitaire sur la littérature \\ et la culture québécoises (CRIILCQ)
}

Les romans de Roger Magini déjouent souvent les frontières entre fiction et réalité, et ce même lorsqu'ils puisent dans l'histoire. Avant de mettre en scène "la momie ventriloque" d'un Lénine nonagénaire qui se serait réveillé d'un coma profond en 1968 dans son dernier roman, Ilitch, mort ou vif (2013), l'auteur d'origine monégasque avait envoyé Gérard de Nerval au Mexique dans Quenamican (2005) tout en déclarant fictionnel le Voyage en Orient de cet auteur français, par exemple.

Son huitième roman, Revoir Nevers ${ }^{2}$ (2006), se distingue toutefois d'un tel rapport contrefactuel à l'histoire, car au lieu de créer une version alternative de la catastrophe nucléaire de Hiroshima, ce court roman commémore cet évènement historique. Afin d'illustrer les difficultés du travail de mémoire, le récit a recours à un dispositif temporel et narratif complexe qui implique fortement le lecteur. En effet, tel un "scénario littéraire" des passages à narration hétérodiégétique qui proposent un projet de livre alternent avec des passages à narration homodiégétique qui semblent mettre ensuite en œuvre ce projet littéraire. Qui plus est, la hiérarchie entre ces deux parties du récit se brouille éventuellement. Cette incongruité sur le plan narratif demande une lecture participative qui rapproche Revoir Nevers des "fictions pour mémoire" telles que les a définies Catherine Dana (1998). Selon Dana, des "romans pour mémoire" se distinguent de simples "romans de mémoire" par "une réflexion menée par l'auteur sur l'écriture de la réalité historique, sur sa mise en forme, sa figuration" (178). Lors du déchiffrement du texte, cette réflexion véhiculée à l'aide de stratégies d'écriture occasionne un "travail de refiguration, [qui] fonctionne comme un appel de mémoire" (16). 
Dans ce qui suit, il s'agira de dégager les réflexions les plus importantes à l'égard d'une appropriation du passé soulevées par l'intrigue de Revoir Nevers, pour ensuite se pencher sur un élément saillant du dispositif narratif du roman, soit les liens intertextuels à La femme des sables (1962) d'Abé Kôbô et au scénario de Hiroshima, mon amour (1960) de Marguerite Duras. ${ }^{3}$ Il s'avérera que grâce au jeu vertigineux de répétition et de superposition textuelle déployé dans Revoir Nevers, ce court roman formule "un appel constant à la mémoire du lecteur” (Dana 16) sous forme fictionnelle.

\section{PARCours COMMÉmoratif-ÉCRIRE MALGRÉ TOUT}

Chargé de rédiger un article commémoratif sur "Hiroshima, 60 ans après", le protagoniste, un journaliste parisien, part pour le Mexique et s'installe à Cholula, une ville choisie au hasard, afin d'y écrire son texte. Une fois arrivé, il éprouve toutefois des difficultés à rendre présent cet épisode douloureux de la Deuxième Guerre mondiale, car et les souvenirs, auxquels il fait appel de manière volontaire, et ceux, qui surgissent telle une affection lors de ses déambulations, lui semblent “dispersés par l'oubli [qui] rédui[...] t sa réflexion à des suites de tableaux incomplets" ( $R N$ 32). Incomplètes et superficielles, ces images mentales lui rappellent la présentation de Cholula dans son guide touristique qui lui fait comprendre le "trop d'oubli" (Ricœur, L'histoire I) autour de lui. De fait, il s'aperçoit du manque total de traces du massacre de 3000 habitants de Cholula survenu en 1519, un évènement qu'il appelle un "minuscule Hiroshima" (RN 33). Suite à ces considérations, c'est à la fin de la première partie du roman que son effort de restituer la catastrophe de Hiroshima par la mémoire se mue en une réflexion plus générale sur l'oubli et sur l'impossibilité de s'approprier :

... le passé, ce quelque chose d'extérieur à moi, parallèle au présent de Cholula, que je revivais comme les retours en arrière d'un vieux film sont faits pour proposer au spectateur une explication du temps (45).

C'est grâce à l'écriture que le journaliste se voit en mesure de s'affranchir de la mainmise d'une suite d'images qui "se déroule à côté de sa propre existence" (45), car l'écriture lui permet de "récupérer [s]on propre temps" (46) en donnant une forme matérielle et, qui plus est, maîtrisée à sa mémoire. Par là, il arrive à conférer une place au passé dans son présent au lieu de s'y perdre comme dans la réalité parallèle d'un “vieux film” (45).

Certes, lors de cette opposition de cinéma et écriture en tant que médias de la mémoire, le narrateur ne compare pas le spectateur au lecteur, 
mais à l'écrivain, juxtaposant ainsi la réception du cinéma et la production de l'œuvre écrite. Néanmoins, ces propos, qui mettent en contraste la "mémoire inerte" (45) au cinéma et la mémoire à l'œuvre qui lie passé et présent lors de l'écriture, ${ }^{4}$ semblent rejoindre les réflexions de Catherine Dana sur la lecture, lorsque celle-ci souligne la nécessité d'“incite[r] le lecteur à un travail de réactualisation des figures qu'elle [la mémoire] met en œuvre dans le texte" (Dana 178). Tout en préférant l'écriture au cinéma, le journaliste ne nie pas les failles de l'appropriation du passé par l'écrit:

Alors même que je savais parfaitement ce qui s'était passé à Hiroshima, je n'ignorais cependant pas que cette tragédie était sans commune mesure avec ce que je pouvais écrire, un passé (fictif ?) dont j'avais perdu les traces [...] (RN 46).

Au lieu de s'attarder sur ces enjeux que constituent la subjectivité de la mémoire et l'impossibilité de s'approprier le passé par les mots, son cheminement le mène ensuite à tourner ses pensées vers le cours de l'histoire qu'il considère être régi par l'oubli. C'est une visite de la pyramide de Cholula qui déclenche une réflexion sur la circularité de l'histoire. Pendant la traversée silencieuse du tunnel qui conduit à la pyramide, l'image des pyramides "qui se sont succédé là, une par-dessus l'autre, la plus importante écrasant toutes les autres, [...], pouvoir sur pouvoir, atrocité sur atrocité" (52), devient une métaphore pour la répétition des inhumanités d'hier, d'aujourd'hui et de demain.

Accompagnant son cheminement, le "silence" du journaliste, répété de manière anaphorique, s'étend pour devenir un "[s]ilence individuel et collectif d'un homme, des hommes, de l'humanité" (54). Dans la troisième partie du roman, une autre anaphore fait écho à ce silence, celle du "jamais plus" (63), que le journaliste martèle comme une incantation pendant la rédaction de son article. Évoquant chaque fois les guerres et les catastrophes du passé et du présent qui l'ont inspirée, il fait toutefois aussitôt mentir cette devise qui constitue la base, non seulement de son article, mais de toute littérature de guerre. ${ }^{5}$ Face à cette impasse, sa réflexion ne peut que se conclure par une question: "Quand célèbrerons-nous la douloureuse victoire de l'homme sur l'inhumain ?” $(68)^{6}$

\section{(R)ÉCRIRE L'HISTOIRE-DÉDOUBLEMENTS TEXTUELS ET MÉMOIRE LITTÉRAIRE}

Le questionnement final sur une fin de l'inhumanité qui régit l'histoire a été inspiré par un roman. Avant de se l'approprier, le journaliste avait d'abord prêté cette réflexion à un personnage de fiction, Niki Jumpei, le protagoniste 
de La femme des sables de l'auteur japonais Abé Kôbô. Quant à Revoir Nevers, le titre est une citation provenant d'un autre prétexte, Hiroshima mon amour (HMA 96). Lançant ainsi un appel à la mémoire littéraire, Revoir Nevers ne se limite toutefois pas à rendre hommage à deux classiques de la littérature mondiale. En entraînant le lecteur dans les dédales d'un puzzle intertextuel, ce court roman se sert des deux prétextes afin d'illustrer la capacité de la littérature de saisir le présent et de ne pas seulement "proposer [...] une explication du temps" (RN 45), mais de la faire vivre au lecteur.

Le premier prétexte, La femme des sables, est matériellement présent dans l'univers fictionnel de Revoir Nevers, car le journaliste relit pendant son vol cette histoire kafkaienne. Qui plus est, il se compare plusieurs fois à l'entomologiste égaré qui cherche refuge chez la femme éponyme du roman dans un village menacé d'être englouti par le sable sans savoir qu'il y sera gardé en captivité afin d'aider les villageois à combattre le sable pour le restant de ses jours (par exemple RN 17,50). Le lien intertextuel, explicitement établi à l'aide d'une note de bas de page (17), incite le lecteur à dresser des parallèles entre les deux univers fictionnels et nourrit ses hypothèses de lecture. ${ }^{7}$ Ce phénomène d'une "superposition de lectures" (Fortier 138), qui ajoute une couche de signification supplémentaire au récit dès lors lu au deuxième degré, met en avant le dilemme du journaliste qui se trouve paralysé entre le devoir de mémoire exprimé par la devise du "jamais plus" et le constat d'une "répétition de l'histoire" dont les cruautés récurrentes semblent aussi inévitables que le sable qui envahit la maison de la Femme des sables. Une telle "lecture relationnelle" souligne alors que tel un pelleteur des sables de la mémoire, le journaliste semble se voir accomplir un travail de Sisyphe.

Si l'interprétation du roman de Kôbô oriente le journaliste dans son parcours de réflexion, le rôle que joue le deuxième prétexte, Hiroshima mon amour, dans la diégèse de Revoir Nevers est plus équivoque. Écrit par Marguerite Duras et mis en scène par Alain Resnais, ce film raconte la brève histoire d'amour d'un Japonais et d'une actrice française de passage à Hiroshima en 1957. Les discussions des amants révèlent leurs passés respectifs_ “"Lui" a perdu toute sa famille dans la catastrophe nucléaire et "Elle" avait été victime de l'épuration dans sa ville natale, Nevers, après la guerre à cause de sa liaison avec un soldat allemand assassiné à la fin de l'Occupation. Ayant survécu à l'humiliation et à la mort de son amour à Nevers, elle est horrifiée de se rendre compte que son amour au Japon sera, lui aussi, éventuellement relégué à l'oubli.

Selon Bernard Pingaud, dans Hiroshima mon amour, Duras et Resnais "superposent deux situations [. . soit la catastrophe individuelle et la 
catastrophe collective] [...], [et ce faisant] ils marquent que cela même que l'on avait cru unique, irrémédiable, peut se répéter" (Le temps 101). De fait, après la résurgence de sa mémoire refoulée, qu'elle vit comme une superposition à son présent au Japon, la Française arrive à raconter le passé, c'est-à-dire à s'en défaire, ôtant ainsi la fascination que le passé conférait à sa situation présente : "Le temps se remet à couler. La mémoire délivrée rend l'héroïne à elle-même, Hiroshima meurt avec Nevers" (Leutrat 87 résumant Pingaud, Premier Plan 5-7). Le protagoniste de Revoir Nevers, quant à lui, constate de manière analogue le cours imperturbable de l'histoire : "Seul subsiste le souvenir et avec lui tout meurt aux portes d'Hiroshima et tout y refleurit, et Cholula avec Hiroshima (RN 54)".

La mise en parallèle des deux textes se justifie par des références extradiégétiques tels le titre déjà mentionné, la citation, “[. . . tu vois, de bien regarder, je crois que ça s'apprend" (HMA 30), placée en exergue du roman ainsi qu'une note de bas de page indiquant que "[s]ont [...] en italiques les citations tirées de Hiroshima mon amour, scénario et dialogue de Marguerite Duras, Gallimard, 1960” (RN 14). De plus, le journaliste puise régulièrement dans les mots du scénario pour exprimer ses réflexions sur Hiroshima. Ainsi, il "sai[t par exemple] qu'Hiroshima se recouvrit de fleurs" ( $R N$ 29, HMA 21 pour la partie en italiques) et se demande comment oublier "la musicienne aveugle aux mains tordues [qui] joue de la cithare" (RN 30, HMA 22 pour la partie en italiques). De manière implicite, le résumé d'un rêve-“On jurerait la Loire. Une jeune femme marche très vite le long de la berge, une bicyclette à la main" (RN 47) — semble décrire la fuite de Nevers de la jeune Française à la fin de la guerre. ${ }^{9}$ Plus directement, une voix non identifiée récite des phrases tirées de Hiroshima mon amour telle une voix hors champ. Ainsi, le journaliste mentionne, par exemple, qu'“[a]vec le vent qui s'est levé une voix a murmuré Du temps passera. Du temps seulement" (RN 64), sans expliquer sa provenance. L'intrusion de cette voix citant les mots de la Française à la gare de Hiroshima (HMA 95) dans le récit du journaliste brise l'illusion mimétique de manière comparable au montage de plans montrant des victimes de Hiroshima pendant une conversation des deux amants (HMA 22). Il s'y ajoute l'effet métatextuel ${ }^{10}$ de la structure narrative du récit qui empêche le lecteur de s'immerger dans l'histoire de Revoir Nevers. La double présence d'un projet littéraire et de son apparente mise en œuvre rappelle, de plus, le mouvement de l'adaptation d'un scénario sur grand-écran et semble, par là, inviter le lecteur à lire ce texte comme la mise en récit d'un autre. 
Sur le plan de l'histoire, une lecture relationnelle révèle d'abord le cheminement intérieur similaire des protagonistes malgré les intrigues divergentes. De fait, le récit scindé en deux de Revoir Nevers raconte le parcours mémoriel du protagoniste, lors duquel il réfléchit au fur et à mesure sur l'insuffisance d'une mémoire apprise, sur sa sélectivité et son surgissement en images médiatisées et disparates, bref sur l'impossibilité de s'approprier le passé. À l'instar de la Française sans nom, il se heurte autant au contraste entre les massacres d'hier et la banalité quotidienne d'aujourd'hui qu'à la conscience de l'impossibilité du combat contre l'oubli, mais à la différence d'elle, il est tout aussi convaincu de l'importance de mener ce combat perdu d'avance.

Il s'avère, dès lors, que la mise en scène de Hiroshima mon amour dans Revoir Nevers ne se limite pas à transposer l'histoire d'une prise de conscience de l'oubli en tant que condition humaine dans un autre contexte, mais la répète avec des différences, ce qui implique un potentiel critique, voire subversif vis-à-vis du prétexte selon Hutcheon (Postmodernism 6) ${ }^{11}$ En effet, tandis que la réflexion sur l'oubli est intrinsèquement liée à une histoire d'amour et, dès lors, située sur le plan personnel dans Hiroshima mon amour, elle est à la fois plus globale et plus abstraite dans le cas du journaliste qui énumère les atrocités passées et présentes dans le monde sans y être personnellement impliqué. Guidé par sa seule conscience et l'amplitude des horreurs qu'il englobe dans son portrait de l'inhumanité de l'homme, il critique implicitement l'oubli collectif de "minuscule[s] Hiroshima[s]" (RN 33) tel le massacre de Cholula, une catastrophe absente de la mémoire collective sélective et hiérarchisée. Cependant ces divergences n'entraînent pas une prise de distance majeure par rapport au prétexte, car Revoir Nevers démontre que l'impression d'une répétition de l'histoire se transpose aisément au présent.

Sur le plan de l'intégration diégétique du prétexte, le statut de Hiroshima mon amour dans l'univers fictionnel de Revoir Nevers est toutefois plus complexe que celui de La femme des sables, qui crée un cadre de réflexion voire d'orientation explicite pour le protagoniste maginien. Tandis que le journaliste fait référence au roman de Kôbô à plusieurs reprises en comparant sa situation à celle de Niki Jumpei, il n'est pas possible de déterminer si ce personnage et narrateur intermittent, qui paraît refaire le parcours de réflexion de la protagoniste durassienne, en est tout à fait conscient, car il n'établit jamais directement le lien au prétexte. De fait, les seuls renvois explicites à l'œuvre Hiroshima mon amour sont le titre, la citation mise en exergue et la note de bas de page qui se situent tous au niveau extradiégétique. 
Au niveau de la diégèse, le journaliste n'indique jamais la provenance des phrases en italiques. Le lecteur est alors amené à se demander s'il cite le prétexte de manière consciente ou s'il s'en sert sans en connaitre l'origine exacte. ${ }^{12} \mathrm{Il}$ en va de même pour les rêves du journaliste qu'il décrit sans les qualifier de restitutions plus ou moins fidèles de scènes de Hiroshima mon amour. L'ingérence d'une voix de provenance inconnue qui prononce les paroles des protagonistes de Hiroshima mon amour (par exemple RN 36 et 64) appuie cette impression d'une présence inconsciente du prétexte. Qui plus est, lors de la seule présence matérielle du film dans la diégèse de Revoir Nevers, le journaliste ne l'identifie pas. Tandis qu'un lecteur qui a vu le film reconnait aisément Hiroshima mon amour lorsque le journaliste décrit "un vieux film en noir et blanc. Musique, générique très sobre puis gros plan de corps enlacés" ( $R N$ 73), diffusé pendant son vol de retour, celui-ci "a oublié le titre du film qu'il n'a pas regardé" (73) à son réveil.

Cette présence ambigüe de Hiroshima mon amour dans la diégèse de Revoir Nevers semble, de la sorte, contribuer de manière implicite à l'opposition de l'écriture et du cinéma quant à leur capacité de transmettre la mémoire déjà brièvement évoquée. Ici, c'est le journaliste qui incarne luimême le spectateur d'un film qui "n'éprouve pas le besoin de se remémorer [et dont] [1]a mémoire est inerte" (45). Par contraste, les liens intertextuels au scénario incitent une lecture au second degré qui oblige le lecteur à s'approprier le texte de manière active au lieu de plonger dans un monde fictionnel parallèle au sien.

De ce fait, même si le lecteur ne connaît pas le prétexte, le dispositif narratif complexe lui permet de suivre un parcours semblable à celui de la protagoniste de Hiroshima mon amour. Celle-ci perçoit le passé dans un présent similaire avant de distinguer les deux dans un mouvement de recul et de s'ancrer, par là, de nouveau dans son présent. À l'aide de la dynamique élaborée d'un récit scindé en deux, Revoir Nevers met en scène une telle transformation d'une superposition répétitive vers la conscience de deux réalités séparées, mais néanmoins liées par le truchement du regard, voire de la lecture.

En guise d'incipit, un narrateur hétérodiégétique, par exemple, annonce que "[l]e livre commencerait par des considérations sur le temps [...]" $(R N$ 13). Cette partie entre crochets est suivie d'une mise en récit de la même scène, mais cette fois-ci du point de vue de l'homme, devenu narrateur homodiégétique, qui affirme que :"J'ai toujours détesté le temps, le temps qui dure et ses petites atrocités" (13). Cette alternance entre un scénario littéraire et les parties qui en constitueraient le texte final n'est toutefois pas constante et le lecteur doit rapidement réviser son hypothèse initiale de 
lecture selon laquelle des segments qui proposent un épisode sont systématiquement suivis par des passages qui mettent en scène la version finale de ce même épisode. D'abord, des ajouts, tel un défilé d'écoliers mis en scène uniquement dans la partie à narration homodiégétique (21), donnent tôt l'impression d'une adaptation très libre. Au fur et à mesure, la frontière entre projet de texte et texte se brouille complètement. ${ }^{13}$

Cette incongruité dans l'architecture de la diégèse oblige le lecteur à la restituer à partir des données des deux niveaux textuels, dont la relation s'avère indécidable. Par conséquent, lors de la refiguration du récit, le lecteur est amené à reconsidérer le rapport des segments textuels à la manière de la Française qui se met à séparer le passé du présent. Le texte ne se présente plus comme un scénario proposé et sa mise en récit finale, mais comme deux récits intercalés procurant des perspectives complémentaires aussi bien qu'enchevêtrées sur une histoire fragmentaire qu'il s'efforce à faire avancer. ${ }^{14}$ De cette manière, la forme du récit qui se braque contre une impression de linéarité, voire de conclusion traduit l'expérience d'écriture du protagoniste. Pour lui, "époques et événements s'entrecroisaient comme la chaine et la trame d'un tissage inachevé" ( $R N$ 67), une image qui décrit aussi la lecture de ce roman lors de laquelle les mémoires littéraire et historique s'entrecroisent en un récit qui raconte "la répétition de l'Histoire ${ }^{15}$ " (67) grâce à celle d'histoires.

\section{Conclusion}

Avec son huitième roman, Revoir Nevers, Roger Magini propose un texte fictionnel, qui non seulement commémore Hiroshima, mais prend comme point de départ le travail de remémoration de cette catastrophe nucléaire afin de mettre en scène, d'un côté, l'appropriation problématique du passé, et de l'autre, une image de l'histoire basée sur l'oubli.

Sur le plan de la diégèse, le roman insiste sur l'accès problématique au passé en soulignant le caractère subjectif des souvenirs. Ensuite, il suscite une réflexion sur les différents moyens de transmission de la mémoire à l'aide d'une mise en contraste du cinéma et de l'écriture. Tout en prenant clairement position dans le champ de l'écriture, le protagoniste souligne qu'une reconstitution du passé par les mots ne peut jamais faire revivre le passé. Inspiré par ses alentours qui lui démontrent l'absence complète du passé sanglant de Cholula dans son présent, il soulève finalement l'enjeu de l'oubli qui est à la base de ce qu'il appelle "la répétition de l'Histoire" (67).

La figure de la répétition semble aussi sous-tendre la diégèse de Revoir Nevers qui se déploie sur fond d'un triple mouvement d'écriture, voire de 
récriture. En effet, l'histoire de Revoir Nevers semble résulter d'une transposition libre des deux intertextes repérés, soit La femme des sables d'Abé Kôbô et Hiroshima mon amour de Marguerite Duras. La mise en récit d'un scénario littéraire constitue le deuxième mouvement. Ce "procès de textualisation" (Marchand 33) comporte toutefois une incongruité, car la logique de répétition ne se maintient pas. Finalement, l'article commémoratif sur la catastrophe de Hiroshima, que rédige le journaliste, représente le troisième mouvement d'écriture. À la différence des deux autres, le lecteur n'a pas accès à ce texte qui n'existe qu'au sein du monde fictionnel de Revoir Nevers. Mettant en avant le processus difficile de rédaction sans en présenter le produit final, le roman souligne, d'une part, l'impossibilité de conclure un projet de commémoration et oblige, de l'autre, le lecteur à refaire le parcours de l'auteur et de s'imaginer le texte commémoratif à partir des données textuelles. Non seulement Revoir Nevers met en scène le "passage de la remémoration personnelle - celle du narrateur ou de l'auteur-à la mémoire collective-formée par les lecteurs" (Dana 40) au sein de la diégèse, mais, qui plus est, le roman dédouble ce mouvement lors du processus de lecture. Ce faisant, Revoir Nevers dépasse le concept d'un "roman pour mémoire" incitant le lecteur à la commémoration d'un événement historique par le truchement d'un effort de refiguration. Il s'agirait alors plutôt d'un "roman pour commémoration", car au lieu de faire restituer le passé au lecteur, il lui fait reconstruire l'effort pour le commémorer, et ce sans oublier le présent. Ainsi, Revoir Nevers peut être lu comme un appel à "bien regarder" le passé tel que le propose la citation tirée de Hiroshima mon amour mise en exergue du roman :

"«ELLE

[...] Par exemple, tu vois, de bien regarder, je crois que ça s'apprend»" (HMA 30).

\section{Notes}

1. Magini 2006, 67.

2. Dans ce qui suit, les références à ce roman seront indiquées par le sigle $R N$ suivi de la page.

3. Le film réalisé par Alain Resnais date de 1957. Marguerite Duras a publié le scénario enrichi d'un synopsis et d'un dossier d'appendices en 1960 chez Gallimard. Désormais, les références à cet ouvrage seront indiquées par le sigle $H M A$ suivi de la page. 
4. Le narrateur ne nie pas la capacité du cinéma de proposer une "explication du temps" (RN 45), un mécanisme, qui a été analysé par Gilles Deleuze dans sa célèbre étude L'Image-Temps (1985). Il la distingue toutefois explicitement de celle de transmettre une expérience temporelle au spectateur. Ce faisant, il convient avec Bernard Pingaud (Le temps 97) qu'un film ne peut que difficilement placer le spectateur dans la situation "d'un homme [ou d'une femme] qui prend conscience de son propre temps", c'est-à-dire d'évoquer ce qu'il appelle "le temps subjectif", même s'il est capable de véhiculer «... une idée du temps que le cinéaste a voulu nous communiquer ".

5. Paul Ricœur (Temps 275) a résumé le rôle de la littérature de guerre comme suit :"[I]l y a peut-être des crimes qu'il ne faut pas oublier, des victimes dont la souffrance crie moins vengeance que récit. Seule la volonté de ne pas oublier peut faire que ces crimes ne reviennent plus jamais."

6. La démarche du journaliste qui s'efforce à exercer un devoir de mémoire face à un avenir menaçant pourrait être lue comme une expression du présentisme tel que l'a défini François Hartog afin de décrire le sentiment d'un double endettement vers le passé et vers le futur qui caractériserait le rapport au temps actuel. Ce faisant, malgré le pessimisme fataliste du journaliste qui a "l'impression d'être l'esclave du passé et d'un avenir qu'il ignore” ( $R N$ 40), dans son cas, “[1]'extension du présent dans la direction du futur [ne] donne [pas] lieu, [. . .] de manière négative, à un catastrophisme (en l'occurrence, pas «éclairé »), [. . .] [mais], positivement, à un travail sur l'incertitude elle-même" (Hartog 268), comme le manifeste son parcours de réflexion, qui accompagne l'acte d'écriture diégétique.

7. De telles références affichées, c'est-à-dire communiquées en tant que telles au lecteur, à un texte précis sont l'un des critères d'une relation intertextuelle intensive selon Manfred Pfister (26-29). Par la note de bas de page, Revoir Nevers ajoute, de plus, un élément autoréflexif, en ce qu'elle thématise la relation intertextuelle. D'après Pfister, celle-ci est d'autant plus intensive, si ces références ne sont pas ponctuelles, mais concernent le texte au complet, de manière à ce que le prétexte sous-tende l'univers fictionnel comme un palimpseste, une description qui correspond au concept d'une transposition hypertextuelle telle que l'a proposée Gérard Genette (237-446). À la différence de Genette, qui effleure seulement la dynamique entre ce qu'il appelle l'hypotexte et l'hypertexte, Pfister met en avant qu'une tension idéologique et sémantique lors de l'intégration du prétexte augmente l'intensité de la relation intertextuelle, car elle suscite des réflexions à l'égard du prétexte. Ainsi, une parodie, définie comme une "repetition with critical difference" par Linda Hutcheon (Postmodernism 6) a un lien intertextuel plus intensif à son prétexte qu'une traduction, par exemple.

8. Gérard Genette (452) a appelé une "lecture palimpsestueuse" ou une "lecture relationnelle [le fait de] lire deux ou plusieurs textes en fonction l'un de l'autre". 
9. Outre ces références se décèlent des parallèles plus allusives. Une description de l'agitation de la main du journaliste juste avant son réveil $(R N 49)$ parait dans ce contexte rappeler "les mains [du Japonais] qui frémissent" (HMA 33) pendant qu'il dort. De plus, en prenant des notes, le journaliste se rend compte d'avoir "fait une faute d'orthographe qu['il] n'a[. . .] pas corrigée immédiatement" (RN 61), en l'occurrence la même faute d'orthographe qu'Alain Resnais a "volontairement laissée" sur une pancarte montrée dans Hiroshima mon amour, comme l'indique une note de bas de page dans le scénario (HMA 55). Sur le plan formel, la division du récit en des segments constituant un scénario littéraire et des segments qui représentent la version finale rappelle, de surcroît, celle du scénario de Hiroshima mon amour, car l'édition indiquée par la note de bas de page comporte une section d'appendices dans laquelle Duras développe des réflexions et remplit quelques ellipses. L'alternance entre une narration hétérodiégétique et une narration homodiégétique dans Revoir Nevers semble répéter ce mouvement de transition entre showing et telling qui s'opère entre le scénario et l'appendice de Hiroshima mon amour.

10. Au sujet de la métanarrativité, voir Nünning.

11. De ce fait, dans la typologie de trois formes de "postmodern rewrites" proposée par Lubomir Doležel (206-207), Revoir Nevers correspondrait le plus à une transposition très libre, c'est-à-dire à un univers fictionnel parallèle à celui du prétexte situé dans un cadre temporel et spatial différent.

12. Au sujet des différentes implications d'une référence thématisée et d'une simple utilisation sur le plan narratif, voir Pfister 26.

13. Voir Marchand pour une analyse très fine de la structure narrative de Revoir Nevers.

14. Marchand (22) souligne, par exemple, que la traversée du tunnel qui constitue une étape majeure du cheminement du protagoniste n'est relatée que dans la partie scénaristique du récit dédoublé. En conférant de la sorte un rôle indispensable dans la dynamique textuelle à la partie scénaristique du récit, le dispositif narratif de ce roman de Magini met en valeur l'œuvre de Marguerite Duras et appelle à sa (re)lecture. En même temps, la structure narrative de Revoir Nevers ne formule pas un plaidoyer implicite pour la priorité de la source, voire de l' "œuvre originale" au détriment de son adaptation. Ce roman hautement intertextuel semble, au contraire, mettre en évidence l'adaptation en tant que moteur de l'histoire littéraire, c'est-àdire démontrer que "stories are born from other stories", comme l'a formulé Linda Hutcheon (Adaptation 2).

15. La personnification du concept de "l'Histoire" à l'aide de la majuscule semble véhiculer le sentiment d'impuissance et de fatalité du narrateur face à un cycle ininterrompu de guerres et d'atrocités qui lui donne l'impression d'une loi naturelle. 


\section{Euvres citées}

Dana, Catherine. Fictions pour mémoire: Camus, Perec et l'écriture de la Shoah. Paris: L’Harmattan, 1998. Imprimé.

Deleuze, Gilles. L'Image-Temps: Cinéma 2. Paris: Minuit, 1985. Imprimé.

Doležel, Lubomír. Heterocosmica: Fiction and Possible Worlds. Baltimore: Johns Hopkins University Press, 1998. Imprimé.

Duras, Marguerite. Hiroshima mon amour. Scénario et dialogues. Paris: Gallimard, 1960. Imprimé.

Fortier, Frances. "Fiction, diction, et d'autres enchantements narratifs." Voix et Images, 31.3 (2006): 134-139. Web. 2 oct. 2013.

Genette, Gérard. Palimpsestes: La littérature au second degré. Paris: Seuil, 1982. Imprimé.

Hartog, François. Régimes d'historicité: Présentisme et expériences du temps, Nouv. éd. augm. et mise à jour. Paris: Seuil, 2012. Imprimé.

Hutcheon, Linda. A Poetics of Postmodernism: History, Theory, Fiction. New York: Routledge, 1988. Imprimé.

Hutcheon, Linda et Siobhan O'Flynn. A Theory of Adaptation. $2^{\mathrm{e}}$ éd. New York: Routledge, 2013. Imprimé.

Kôbô, Abé. La femme des sables. $2^{\mathrm{e}}$ éd. Traduit par Georges Bonneau. Paris: Stock, 1990. Imprimé.

Leutrat, Jean-Louis. Hiroshima mon amour. $2^{e}$ éd. Paris: Armand Colin, 2008. Imprimé.

Magini, Roger. Ilitch, mort ou vif. Paris: Grasset, 2013. Imprimé.

- Quenamican. Montréal: Pleine Lune, 2005. Imprimé.

- Revoir Nevers. Montréal: Pleine Lune, 2006. Imprimé.

Marchand, Marc-André. Des singularités narratives comme manifestations d'une problématisation de l'autorité narrative chez Magini, Yergeau et Soucy: Enjeux et jeux d'écriture dans trois romans contemporains Québécois. Mémoire de maitrise. Université du Québec à Rimouski, 2011. Imprimé.

Nünning, Ansgar. "Metanarration als Lakune der Erzähltheorie. Definition, Typologie und Grundriß einer Funktionsgeschichte metanarrativer Erzähleräußerungen." Arbeiten aus Anglistik und Amerikanistik. 26.2 (2001): 125-164. Imprimé.

Pfister, Manfred. "Konzepte der Intertextualität.“ Intertextualität. Ulrich Broich et Manfred Pfister (dir.). Tübingen: Niemeyer, 1985. 1-30. Imprimé.

Pingaud, Bernard (dir.). Premier Plan: Hommes, auvres, problèmes du cinéma. 18, oct. (1961). Imprimé.

- "Le temps: Dialectique de la mémoire et de l'oubli." "Tu n'as rien vu à Hiroshima!" Un grand film Hiroshima mon amour. Raymond Ravar (dir.). Bruxelles: Éditions de l'Institut de Sociologie, 1962. 89-111. Imprimé.

Ricœur, Paul. La mémoire, l'histoire, l'oubli. Paris: Seuil, 2000. Imprimé.

. Temps et récit; Le Temps raconté.Vol. 3. Paris: Seuil, 1985. Imprimé. 